\title{
Aggresomes predict poor outcomes and implicate proteostasis in the pathogenesis of pediatric choroid plexus tumors
}

\author{
Nada Amer ${ }^{1} \cdot$ Hala Taha ${ }^{2,3}$. Dina Hesham ${ }^{1} \cdot$ Nouran Al-Shehaby $^{1} \cdot$ Amal Mosaab $^{1} \cdot$ Mohamed Soudy $^{4} \cdot$ Aya Osama $^{4}$. \\ Noura Mahmoud ${ }^{5} \cdot$ Moatasem Elayadi $^{5,6} \cdot$ Ayda Youssef $^{7,8} \cdot$ Mohamed Elbeltagy $^{9,10}$. Mohamed Saad Zaghloul ${ }^{11,12}$. \\ Sameh Magdeldin ${ }^{4,13}$ (1) Ahmed A. Sayed ${ }^{14,15} \cdot$ Shahenda El-Naggar ${ }^{1}$ (i)
}

Received: 28 November 2020 / Accepted: 31 December 2020 / Published online: 26 January 2021

(c) The Author(s) 2021

\begin{abstract}
Purpose Protein misfolding and aggregation result in proteotoxic stress and underlie the pathogenesis of many diseases. To overcome proteotoxicity, cells compartmentalize misfolded and aggregated proteins in different inclusion bodies. The aggresome is a paranuclear inclusion body that functions as a storage compartment for misfolded proteins. Choroid plexus tumors (CPTs) are rare neoplasms comprised of three pathological subgroups. The underlying mechanisms of their pathogenesis remain unclear. This study aims to elucidate the prognostic role and the biological effects of aggresomes in pediatric CPTs. Methods We examined the presence of aggresomes in 42 patient-derived tumor tissues by immunohistochemistry and we identified their impact on patients' outcomes. We then investigated the proteogenomics signature associated with aggresomes using whole-genome DNA methylation and proteomic analysis to define their role in the pathogenesis of pediatric CPTs.

Results Aggresomes were detected in $64.2 \%$ of samples and were distributed among different pathological and molecular subgroups. The presence of aggresomes with different percentages was correlated with patients' outcomes. The $\geq 25 \%$ cutoff had the most significant impact on overall and event-free survival ( $p$-value $<0.001$ ) compared to the pathological and the molecular stratifications.

Conclusions These results support the role of aggresome as a novel prognostic molecular marker for pediatric CPTs that was comparable to the molecular classification in segregating samples into two distinct subgroups, and to the pathological stratification in the prediction of patients' outcomes. Moreover, the proteogenomic signature of CPTs displayed altered protein homeostasis, manifested by enrichment in processes related to protein quality control.
\end{abstract}

Keywords Aggresomes $\cdot$ Choroid plexus $\cdot$ Proteogenomics $\cdot$ Proteostasis $\cdot$ Prognosis

\section{Introduction}

Choroid plexus tumors (CPTs) are rare intraventricular neoplasms that account for $0.2 \%$ to $0.4 \%$ of all central nervous system neoplasms, with up to $20 \%$ during the first year of life [1]. CPTs are pathologically classified into three subgroups; benign choroid plexus papilloma (CPP, WHO grade I), intermediate atypical CPP (ACPP, WHO grade II), and the aggressive malignant choroid plexus carcinoma (CPC,

Supplementary Information The online version contains supplementary material available at https://doi.org/10.1007/s1106 0-020-03694-3.

Shahenda El-Naggar

shahenda.elnaggar@57357.org

Extended author information available on the last page of the article
WHO grade III) that is associated with poor prognosis [2]. CPPs have a favorable prognosis after surgical resection and rarely require additional treatment [3], while CPCs usually require surgical removal with adjuvant chemotherapy and radiotherapy [3]. The molecular stratification of pediatric CPTs identified two subgroups that distinguished high-risk patients regardless of their pathological classification $[4$, 5]. Current evidence supports the involvement of TP53 [4], Notch signaling [6], and Sonic Hedgehog (SHH) [7] in the pathogenesis of these tumors; however, the underlying etiology is yet to be identified.

The cellular proteome is a highly complex system that requires the coordination of protein quality control (PQC) machinery to balance protein synthesis, folding, and degradation [8]. Continual proteotoxic stress imposed by an imbalance in protein levels or protein aggregation 
causes cells to compartmentalize misfolded/ aggregated proteins into distinct quality control compartments [8]. The aggresome is a single juxtanuclear inclusion body wherein misfolded proteins are delivered by the dyneinmediated retrograde transport and ensheathed by the intermediate filament vimentin [9]. Aggresome formation has emerged as a drug resistance mechanism to overcome proteotoxic stress caused by proteasome inhibition-based therapy in selective tumors such as multiple myeloma [10, 11], pancreatic cancer [12], breast cancer [13], and lymphoma [10]. We have previously identified aggresomes in pediatric CPTs [14] and pediatric medulloblastoma (MB) [15]. They were inherently present in both tumors before therapy and were associated with poor outcomes in the non-WNT/SHH molecular subgroup of MB [15], suggesting that they provide a survival advantage to these tumors.

In the current study, we report the role of aggresomes as a prognostic molecular marker in pediatric CPTs. Additionally, we explore the molecular signature of aggresomepositive CPTs using genome-wide methylation profile in correlation to cellular proteome which implicates altered proteostasis in the pathogenesis of pediatric CPTs.

\section{Materials and methods}

\section{Patient and tissue samples}

CPT samples were retrospectively collected from the Pathology Department at the Children's Cancer Hospital Egypt 57357 (CCHE) after the approval of the Institutional Research Ethics Board (IRB) for waiver of consent. None of the patients had previously received chemotherapy or radiotherapy. Histopathologic review of all CPT samples was performed according to the WHO guidelines. Our cohort was comprised of 23 patient formalin-fixed-paraffin embedded (FFPE) tissue samples, 2 fresh frozen (FF), and 17 patients represented by both FFPE and their matched FF tissue samples. Treatment protocol of CPT patients was adopted from CPT-SIOP-2009 study [16].

\section{Immunohistochemical analysis (IHC)}

IHC was performed using the Ventana Benchmark XT automated system (Ventana Medical System). Antibodies against the following antigens were used: TP53 (Clone DO-7, N1581, Dako; ready to use), vimentin (Ventana 790-2917; dilution 1:100), and pan-keratin (Ventana 7602595; dilution 1:100). Aggresome-positivity was considered only for cells exhibiting juxtanuclear staining of vimentin.

\section{Sample processing and the Infinium methylation EPIC array}

Genomic DNA was extracted from FFPE tumor samples using QIAamp DNA FFPE tissue kit (Qiagen) and FF tumor samples using the Gene JET genomic DNA (Thermo Fisher) according to the manufacturer's instructions. DNA was quantified using the DENOVIX Fluorometer (ds DNA High Sensitivity). The quality of the extracted FFPE DNA samples was assessed by Illumina FFPE QC kit (Illumina Inc.). Bisulfite conversion of extracted DNA was performed using the EZ DNA methylation kit (D5002, Zymo Research) according to the manufacturer's instructions using the alternative incubation conditions recommended for the Illumina Infinium methylation arrays. Bisulfiteconverted FFPE DNA was then restored with Infinium HD FFPE DNA Restore Kit (WG-321-1002, Illumina Inc.). Restored bisulfite-modified DNA samples were hybridized to the Illumina Infinium Human Methylation EPIC bead chips and scanned using the Illumina iScan microarray scanner according to the manufacturer's recommendations (Illumina Inc.). Methylation data are available through Gene Expression Omnibus (GEO: http://www.ncbi.nlm. nih.gov/geo/), accession number GSE156090.

\section{Methylation data analysis}

Methylation analysis was performed using R statistical language v.3.5.2. Raw signal intensities were obtained from Illumina intensity data (IDAT) files using the minfi Bioconductor package v. 1.29.3 [17]. Samples quality control steps were performed including; sample swap using the pairwise comparison of 59 genotyping probes and detection of $p$-value. Samples with $p$-value $>0.01$ $(\mathrm{n}=2)$ were excluded from further analysis. Each sample was individually normalized using functional normalization (FunNorm) [18]. Batch effect prediction was done by singular value decomposition (SVD) using the ChAMP Bioconductor package v.3.10 [19], subsequent correction for the type of tumor samples (FFPE or FF) was performed by ComBat algorithm [20]. Probes failed in the detection of $p$-value $>0.01$ in at least one sample $(n=91,679)$ and probes located on sex chromosomes $(n=15,413)$ were removed. Probes containing SNPs $(n=24,747)$ in their bodies, or at a $\mathrm{CpG}$, or single base extension site (SBE) with minor allele frequency (maf) $\geq 0.01$, were excluded and those showing cross-reactivity $(\mathrm{n}=38,077)[21$, 22] were eliminated. Beta $(\beta)$ and $M$ values were generated from the remaining probes $(n=696,293)$. The most variably methylated probes $(n=36,279)$ were selected based on the standard deviation ( $\mathrm{SD}>0.7)$. The $\mathrm{t}$-distributed 
stochastic neighbour embedding (t-SNE) analysis was performed using Rtsne package v.0.11 [23] with non-default parameters [theta $=0, \mathrm{pca}=\mathrm{F}$, max_iter $=2000]$ based on pairwise Pearson's correlation. Unsupervised hierarchical clustering was done using Euclidean distance and ward. D2. The next-generation molecular neuropathology (MNP) platform was used to check the methylation-based classification of our CPT samples [24]. All duplicates samples $(n=17)$ were removed from any further analysis. Differentially methylated positions (DMPs) were identified using champ.DMP function from ChAMP Bioconductor package v.3.10 [25] at the significance of adjusted $p$-value $<0.05$. Differentially Methylated Regions (DMRs) were identified by Bumphunter with default settings [26]. Circos plot of DMRs was performed by Circlize package v0.4.8 with default parameters [27]. Three stratification approaches of CPTs (methylation-based classification, aggresomepositivity, and the $\geq 25 \%$ aggresome cutoff) were used to examine the correlation of aggresomes with methylation signature.

\section{Methylation data GO and KEGG enrichment analyses}

The gene lists derived from DMPs with adjusted $p$-value $<0.05$ and $\log$ FC $(1.5,-1.5)$ were used for gene set enrichment analysis using ToppFun in the ToppGene suite [28]. Genomic Regions Enrichment of Annotations Tool v3.0.0 (GREAT) was utilized for the gene ontology (GO) enrichment analysis of the DMRs using default settings [29]. Bed files denoting the start, the end positions of the DMRs, as well as the chromosome numbers were uploaded and mapped against the hg 19 human reference genome. Network analysis of the DMPs' genes was performed by NetworkAnalyst 3.0 using STRING interactome with 700 confidence score cutoff, and experimental evidence criterion was required [30]. Analyses for enriched Kyoto Encyclopedia of Genes and Genomes (KEGG) pathways of those networks were performed. Only terms with false discovery rate (FDR) less than 0.05 using the Benjamini-Hochberg (BH) method were considered significant for all analyses.

\section{Survival analysis}

All statistical analyses were performed using R statistical environment v3.3.2. Overall survival (OS) was calculated from the initial diagnosis to last follow-up or death due to disease. Event-free survival (EFS) was calculated from the initial diagnosis to the time of an event. An 'event' was defined as tumor progression, recurrence or death. OS and EFS were estimated by the Kaplan-Meier method, and differences between groups were assessed by the log-rank test. Survival estimates referred to 2 years from diagnosis and the related $95 \%$ confidence intervals $(95 \% \mathrm{CI})$ were calculated. One patient died shortly after surgery before assigning any protocol and was removed from the analysis.

\section{Protein extraction}

Twenty one frozen samples were collected and sample sections were homogenized in urea extraction buffer $(8 \mathrm{M}$ urea, $500 \mathrm{mM}$ Tris- $\mathrm{HCl} \mathrm{pH} 8.5$, and protease inhibitors) using Dounce homogenizer. Lysates were incubated at room temperature for $1 \mathrm{~h}$ before centrifugation at $10,000 \mathrm{rpm}$ for $30 \mathrm{~min}$. Supernatants containing extracted proteins were collected and proteins were quantified using the Pierce BCA protein assay kit (23225, Thermo Fisher).

\section{In-gel digestion}

Forty micrograms of proteins from each sample were separated using $12 \%$ SDS-PAGE. Proteins were fixed by adding a fixing solution (50\% methanol and $12 \%$ acetic acid) with overnight incubation at $4{ }^{\circ} \mathrm{C}$. Gel pieces were washed using gel-wash buffer $(50 \%$ acetonitrile in $50 \mathrm{mM}$ ammonium bicarbonate) and dried using speed vacuuming. Reduction buffer $(10 \mathrm{mM}$ dithiothreitol in $50 \mathrm{mM}$ ammonium bicarbonate) was added to dried gel pieces and incubated at $60^{\circ} \mathrm{C}$ for $30 \mathrm{~min}$. Alkylation buffer $(55 \mathrm{mM}$ iodoacetamide in $50 \mathrm{mM}$ ammonium bicarbonate) was then added and incubated in dark at room temperature for $30 \mathrm{~min}$. Gel pieces were washed using $25 \mathrm{mM}$ ammonium bicarbonate before adding acetonitrile for $15 \mathrm{~min}$. Digestion solution $(10 \mathrm{ng} / \mu \mathrm{L}$ trypsin in $25 \mathrm{mM}$ ammonium bicarbonate) was added to gel pieces until gel hydration and incubated overnight at $37^{\circ} \mathrm{C}$. Extraction buffer (66 acetonitrile: 33 milliQ water: 1 formic acid) was then added to extract digested peptides.

\section{LC-MS/MS}

The nanoflow reverse-phase liquid chromatography (LC) followed by mass spectrometry (MS/MS) analysis was carried out using Triple TOF $5600+$ (AB SCIEX) interfaced at the front end with an Eksigent nano-LC 400 auto-sampler with an Ekspert nano-LC 425 pumps. Samples were automatically injected into a peptide trap column Chrome XP; C18-CL, 5 um (Chrome XP; C18-CL, 0.5 mm I.D. $\times 10 \mathrm{~mm}$, 5 - $\mu \mathrm{m}$ particle size, 120 - $\AA$ pore size; SCIEX). The MS and MS/MS ranges were $400-1250 \mathrm{~m} / \mathrm{z}$ and $170-1500 \mathrm{~m} / \mathrm{z}$, respectively. A 55-min linear gradient of $3-40 \%$ solution B ( $80 \%$ acetonitrile, $0.2 \%$ formic acid) was applied. The mass spectrometry proteomics data have been deposited to the ProteomeXchange Consortium via the PRIDE [1] partner repository with the dataset identifier PXD021076 and 10.6019/PXD021076. RAW files were converted into 
mascot generic format (mgf) using AB-SCIEX MS data converter v.1.3 and searched using $X$ !Tandem in peptide shaker v. 1.16.38 against human UniProtKB/Swiss-Prot database (2018 release; 173361 proteins). Proteins identified with a minimum of one unique peptide and a minimum of two confident spectra were selected for further statistical analyses.

\section{Proteomics data analysis}

Twenty one exported CSV files containing Uniprot accession number and label-free protein abundance based on normalized spectral abundance factor (NSAF) were merged into a single file using ProteoSelector (www.57357.org/proteomics -unit). A sanity check was performed to evaluate the accuracy of the sample, class labels, and data structure. Then the data imputation was conducted based on the minimum NSAF protein value [31] followed by the filtration process based on non-relative standard deviation (NRSD) [32]. Samples were then normalized using probabilistic quotient normalization (PQN) [33] then log-transformed, and autoscaled. Data was subjected to unpaired t-test and the significant output was considered only when $p$-value $\leq 0.05$. The significant differentially expressed proteins (DEPs) were analyzed using UniProtR [34]. The biological processes, molecular functions, and cellular components of identified protein were obtained by ToppFun in the ToppGene suite [28]. Protein-protein interactions (PPI) and network analyses were carried out by Cytoscape [35].

\section{Results}

\section{Aggresome predicts poor prognosis CPT patients}

The clinicopathological characteristics of all patients are summarized in (Supplementary Table 1). Methylation EPIC array was used to molecularly stratify CPT samples (Fig. 1a). Sample swap affirmed the concordance between matched FFPE and FF tissue samples (Supplementary Fig. 1a). Two samples were excluded from further analysis based on a high $p$-value compared to background signals of control probes (Supplementary Fig. 1b). Technical batch effects were excluded by singular value decomposition (SVD) (Supplementary Fig. 1c). Normalized and filtered data of 57 tumor samples were used for the selection of the most variable methylated probes $(n=36,279)$. These probes were distributed across all chromosomes and different genomic features, with the majority in the open sea and gene bodies (Supplementary Fig. 1d). Subsequent t-SNE analysis and hierarchical clustering across the dataset using most variable probes identified two distinct groups with all paired samples of FFPE and FF clustered together (Fig. 1b and Supplementary Fig. 2). Methylation cluster "A" comprised 19 CPPs (63.33\%) and 11 ACPP (36.67\%.) with no CPC samples. In contrast, cluster "B" contained all CPC samples $(\mathrm{n}=14,51.8 \%), 3$ CPPs $(11.11 \%)$, and 10 ACPP (37.09\%). The MNP classifier was used to validate the methylation-based classification of CPT samples (Fig. 1c and Supplementary Table 2).

IHC identified aggresomes in $64.2 \%$ of samples $(n=27)$ distributed among all pathological and molecular subgroups. The cytokeratin-positive paranuclear stain was detected in most of the samples, however; its percentage did not coincide with the level of vimentin (Fig. 2a and Supplementary Table 2). Aggresomes-based classification identified two groups, where aggresome-positive tumors comprised all CPCs $(n=12)$, ACPPs $(n=10)$, CPPs $(n=5)$, and $77.7 \%$ of cluster "B" tumors $(n=21)$. On the other hand, CPPs $(n=10)$, ACPPs $(n=5)$, and $71.4 \%$ of cluster "A" tumors $(\mathrm{n}=15)$ fell in the aggresome-negative group (Fig. 2b). The overexpression of TP53 was observed in $26.19 \%$ of all tumors $(\mathrm{n}=11)$. TP53-positive tumors all had aggresomes, fell in cluster "B" and distributed among all pathological subgroups (Supplementary Table 2). TP53 had a significant impact on both OS and EFS ( $p$-value $=0.03$ ) (Supplementary Fig. 3). In addition, the proliferation indices of Ki-67/ MIB-1 with $\geq 30 \%$ cutoff aslo had a significant effect on OS and EFS; $p$-value $<0.0001$ ) (Supplementary Table 3). Survival analysis using different percentages of aggresomes ranging from 10 to $30 \%$ was performed to test the impact of aggresome on patient outcome compared to pathological and molecular classifications, and TP53 status (Supplementary Table 4 and Supplementary Fig. 3). Aggresome-positivity correlated significantly with patients' outcomes and maintained significance at all proposed cutoffs. The $\geq 25 \%$ aggresomes cutoff had the highest impact on both OS and EFS ( $p$-value $<0.001$ ) (Fig. 2c). Analysis of clinical variables including age at diagnosis (OS $p$-value $=0.53$ and EFS; $p$-value $=0.54)$, and extent of resection (OS and EFS; $p$-value $=0.97$ ) had no significant effect on outcomes (Supplementary Table 3).

\section{DNA methylation signature implicates proteostasis in the pathogensis of CPTs}

Statistically significant DMPs of the three stratification approaches were identified (Supplementary Tables 5 and 6) and used for gene set enrichment analysis (GSEA) (Supplementary Table 7). Methylation-based classification using DMPs with adjusted $p$-values $<0.05$ and $\log$ FC $(1.5,-1.5)$ identified 1840 genes that showed enrichment in developmental processes like regulation of cell morphogenesis, neuron development and generation in addition to regulation of protein localization, and cytoskeleton protein 
a

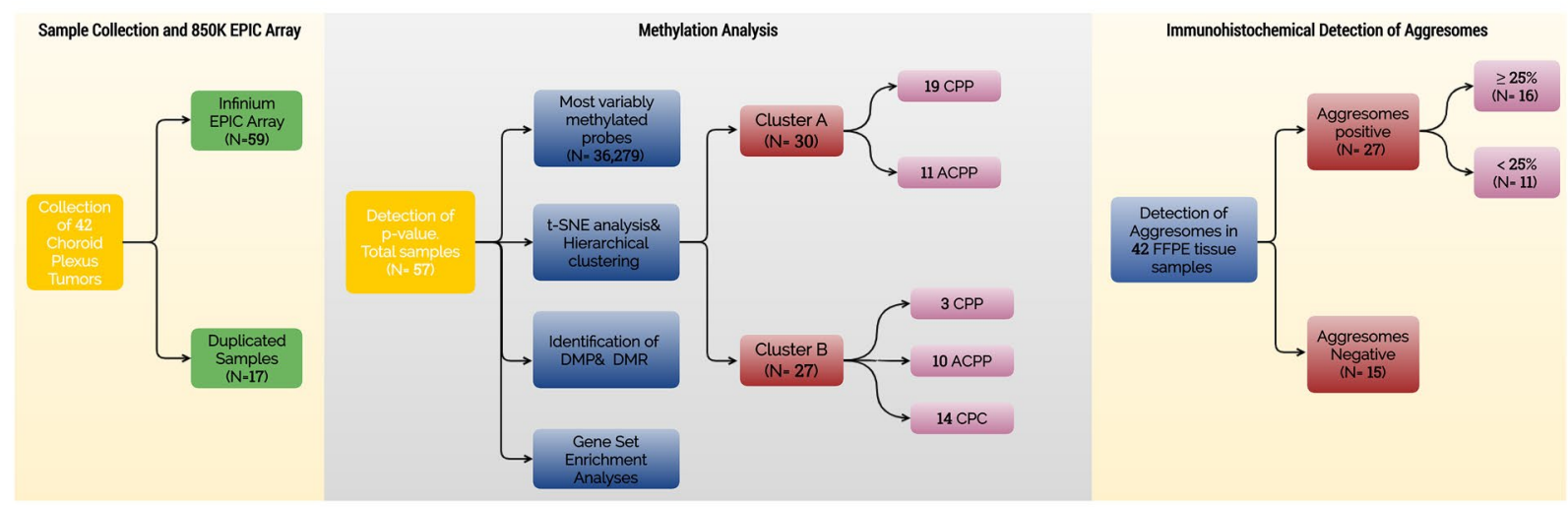

b

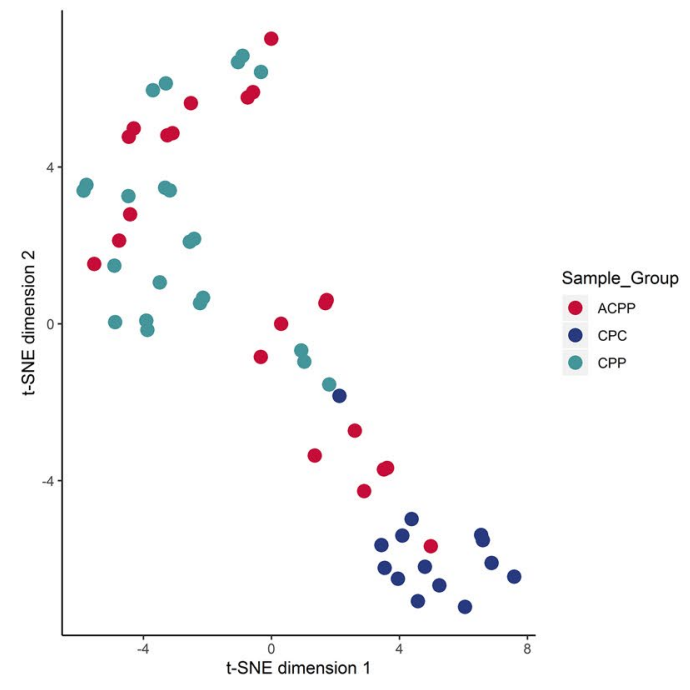

C

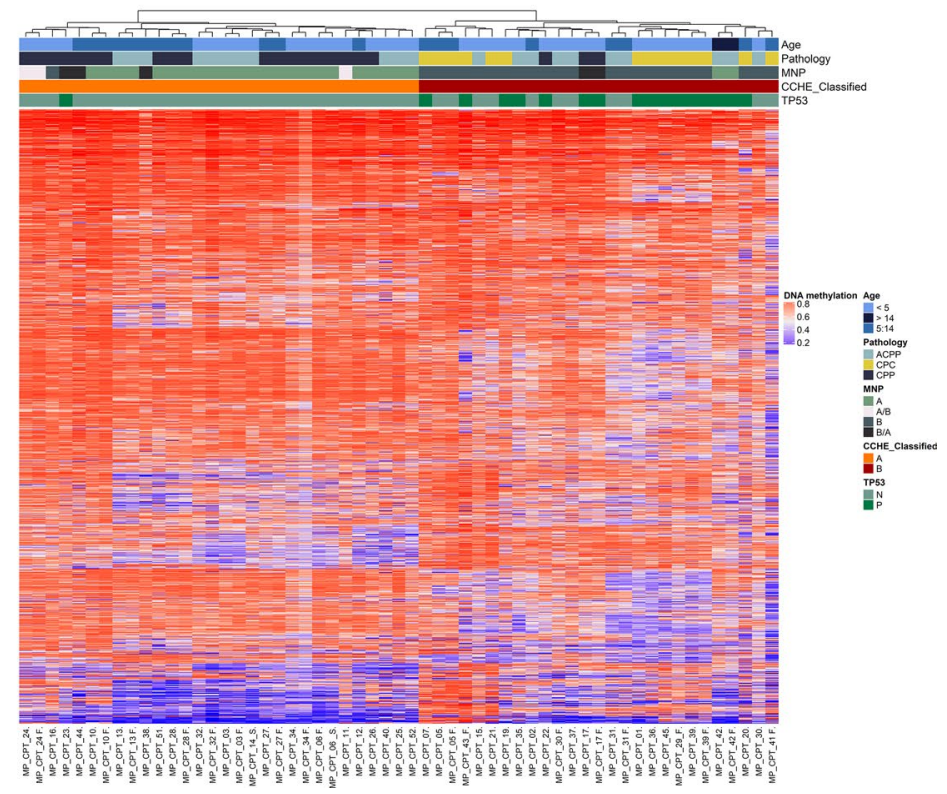

Fig. 1 Methylation-based classification of pediatric CPTs identified two molecular subgroups. a Overview flow chart of sample population and analyses. $b$ t.SNE plot of the CPT dataset $(n=57)$ using the most variable methylated probes $(n=36,279)$, showing two distinct

binding organization (Fig. 3a and Supplementary Fig. 4a). Aggresome-positivity identified 358 genes that had a functional enrichment of protein binding and microfilament motor activity, as well as intracellular protein transport and localization (Fig. 3b and Supplementary Fig. 4b). Furthermore, the $25 \%$ aggresomes cutoff comparison identified 1243 genes enriched in developmental processes, protein binding, voltage-gated ion channel, and protein localization (Fig. 3c and Supplementary Fig. 4c).

PPI network of aggresome-positivity was included within the methylation-based classification network (Fig. 3d, e, f, and Supplementary Table 8). The PPI networks of the molecular entities compared to pathological classification. c Heatmap of $\beta$-values of the most variable methylated probes after the assignment of CPTs subgroups into clusters "A" and "B", combined with age, pathology, MNP classification, and TP53 status

differentially methylated genes of the three comparisons were enriched in the mTOR signaling pathway, MAPK, autophagy, apoptosis, and PI3K-Akt signaling pathway, mitophagy, pancreatic, prostate, and breast cancers in addition to chronic myeloid leukemia pathways (Fig. 3g, h, and i). Despite having the lowest number of genes in the PPI subnetwork, aggresomes positivity identified the highest number of enriched pathways with a $30.8 \%$ concordance with methylation-based classification (Supplementary Fig. 4d).

DMRs of the three stratification approaches displayed a similar pattern across all chromosomes (Fig. 4a and Supplementary Table 9). ZICI and ZIC4 genes were 
a

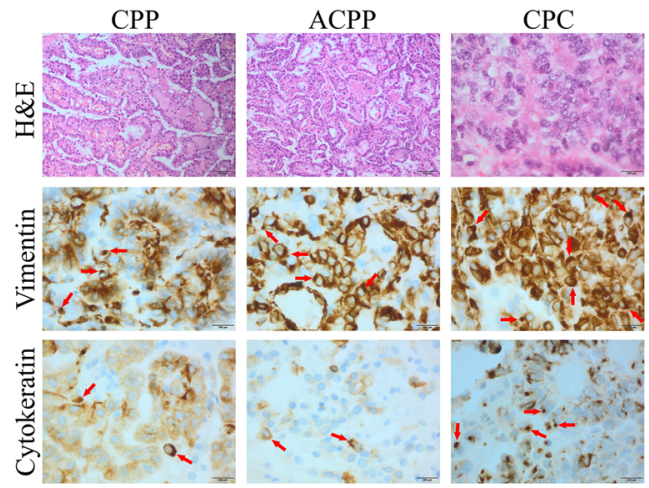

b

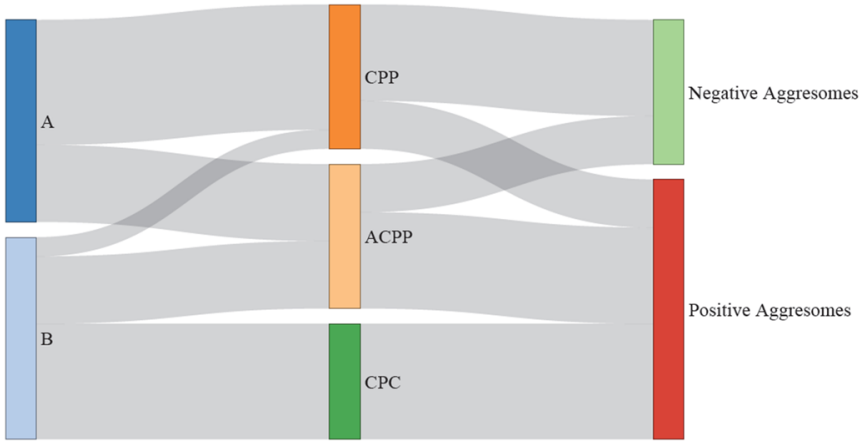

C
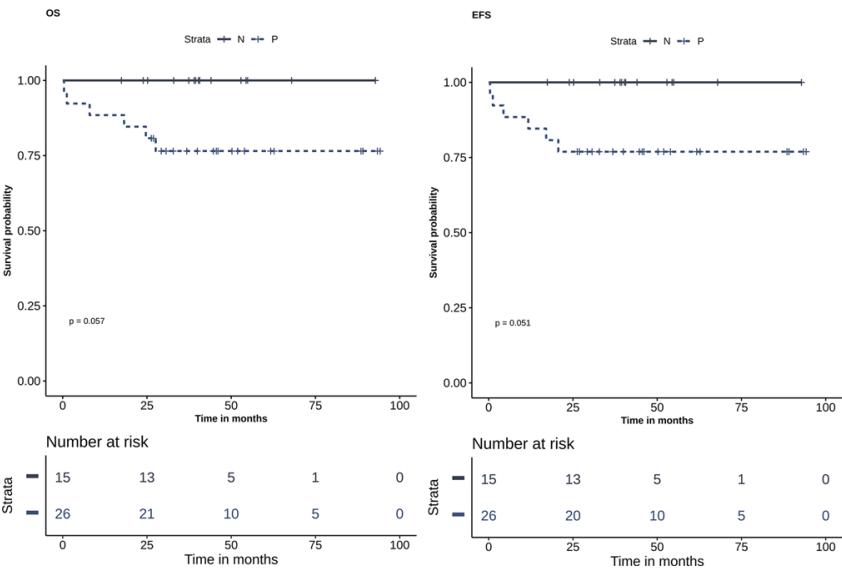

Fig. 2 Characterization of aggresomes in CPTs and their association with the clinical outcomes. a Hematoxylin and eosin (H\&E) staining of CPP, ACPP, and CPC FFPE tissues and IHC analysis of vimentin and cytokeratin. Juxtanuclear dot-like staining of vimentin and cytokeratin were detected in all pathological subgroups with different percentages. b Distribution of aggresomes among different pathologi-

identified in the top significant DMRs in the three stratification approaches ( $p$-values $<0.000)$. DMPs that annotated to those genes were found in the body and promoter regions and were hypermethylated in all positions and all comparisons. The GO analysis of DMRs mainly showed enrichment in developmental processes (Fig. 4b and Supplementary Table 10). Furthermore, pathway enrichment analysis identified, the intrinsic apoptotic signaling pathway by p53 class mediator pathway, and the ATF6-mediated unfolded protein response (Supplementary Table 10).

\section{Proteomics support DNA methylation signature in CPTs}

A total of 2147 proteins were identified from all samples, filtered into 784 non-redundant proteins for subsequent normalization and differential analysis (Supplementary
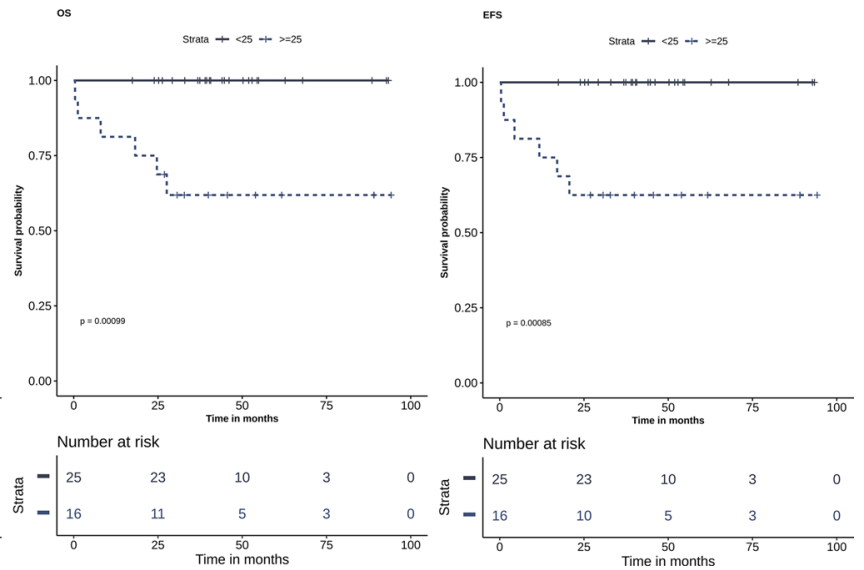

cal and molecular subgroups; NetworkD3 and dplyr packages were used to obtain the Sankey plot. c Kaplan-Meier plot of overall (OS) and event-free survival (EFS) for 41 CPT patients. Survival analysis was separated by aggresome-positivity (positive vs. negative), and the $\geq 25 \%$ aggresomes cutoff. $P$-values were calculated using the logrank test

Table 11). DEPs among the three stratification approaches were identified (Supplementary Fig. 5a, b, c, and Supplementary Table 12) and segregated CPT samples into two distinct subgroups using partial least square regression analysis (PLS) (Supplementary Fig. 5d, e, and f). Hierarchical clustering using the top 50 DEPs showed a clear separation between samples specifically within the aggresome-positivity stratification (Fig. 5a, b, and c).

The GO analysis of the DEPs derived from methylation-based classification showed enrichment in terms of protein localization and transport, chaperone-mediated autophagy, unfolded protein response, RNA binding, and lysosomal degradation (Fig. 5d, Supplementary Table 13). DEPs of aggresome-positivity showed enrichment in the cellular response to unfolded protein along with chaperone binding, microtubule-based movement, and intermediate filament remodeling (Fig. 5e, Supplementary 
a

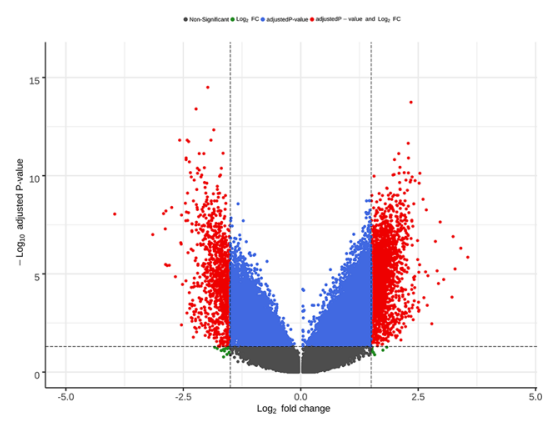

b

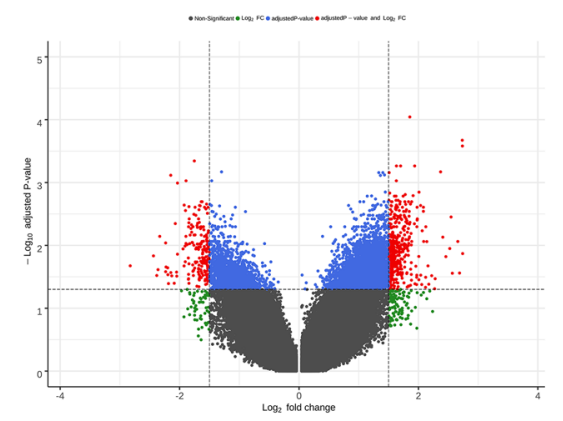

e

d
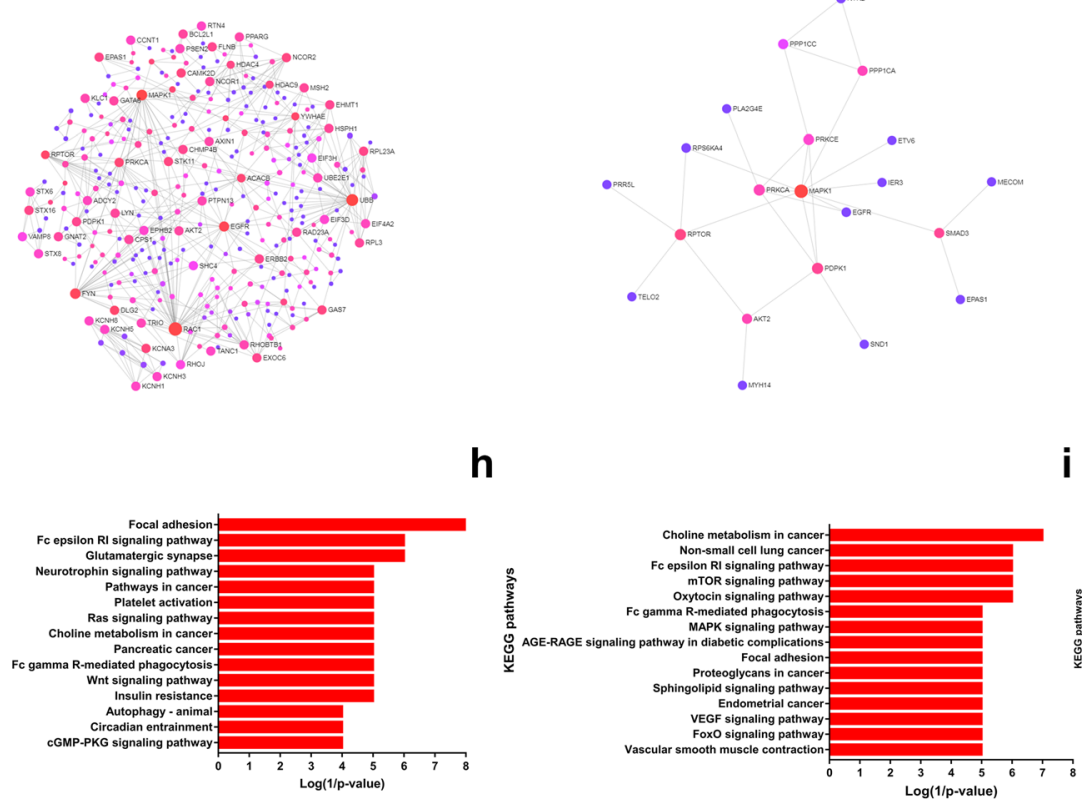

C

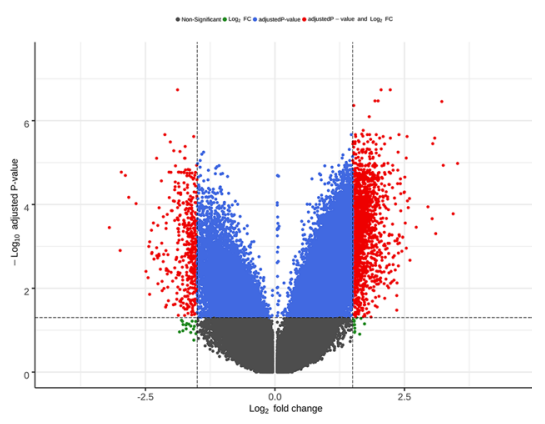

$\mathbf{f}$

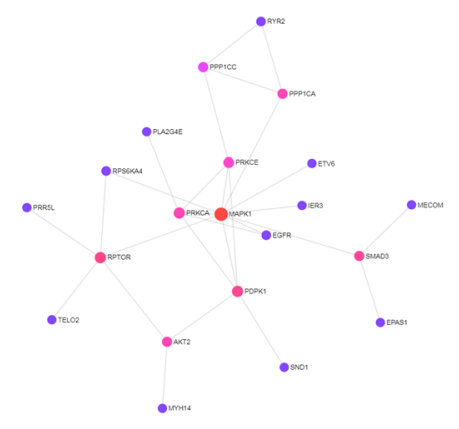

g

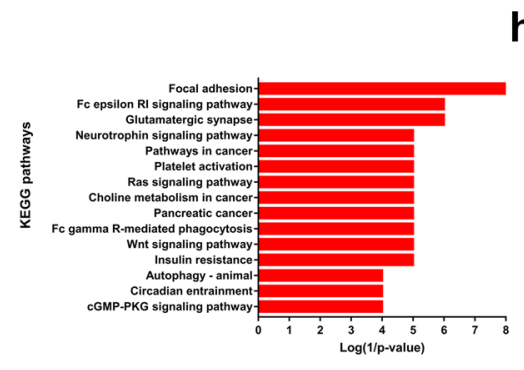

$\mathbf{h}$

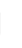

Fig. 3 Enrichment analysis of DMPs. Volcano plot of the DMPs highlighting the hypomethylated and hypermethylated genes in a methylation-based classification, b aggresomes-positivity, and c the $\geq 25 \%$ aggresomes cutoff, with $\log 2$ FC on the $\mathrm{x}$-axis and $\log 10$ adjusted $p$-value on the y-axis. The horizontal line represents the cutoff of the adjusted $(p$-value $<0.05)$ and the vertical lines represent the cutoff of the $\log 2$ FC (1.5 and -1.5). PPI network analysis of significant DMPs of $\mathbf{d}$ methylation-based classification, e aggresomes-positivity, and (f) the $\geq 25 \%$ aggresomes cutoff. Enrichment analysis of KEGG pathways in $\mathbf{g}$ methylation-based classification, $\mathbf{h}$ aggresomespositivity, and (i) the $\geq 25 \%$ aggresomes cutoff with enriched pathways plotted on the $\mathrm{y}$-axis versus their $\log (1 / p$-values $)$ on the $\mathrm{x}$-axis
Table 13). Finally, DEPs based on the $25 \%$ aggresomes cutoff showed enrichment in cellular processes involved in late endosomal microautophagy, chaperone-mediated autophagy, lysosomal degradation, and calcium homeostasis (Fig. 5f, Supplementary Table 13). The PPI network of DEPs in the three stratifications were found to be differentially methylated genes and related to protein regulation machinery (Fig. $5 \mathrm{~g}$, h, and i).

\section{Discussion}

In this study, we assessed the prognostic impact of aggresomes in pediatric CPTs and examined their associated proteogenomic signatures. CPT patients were molecularly stratified using genome-wide methylation profiling. Consistent with previous reports $[4,5]$ the methylation-based classification of pediatric CPTs identified two molecular subgroups with all CPCs clustered in 
Fig. 4 DMRs and their genomic context. a Circos plot of DMRs for the 3 comparisons and their distributions across chromosomes. b GO analysis of top significant $(\mathrm{FDR}<0.05)$ biological processes, molecular functions, and cellular components of DMRs in all each stratification approaches a

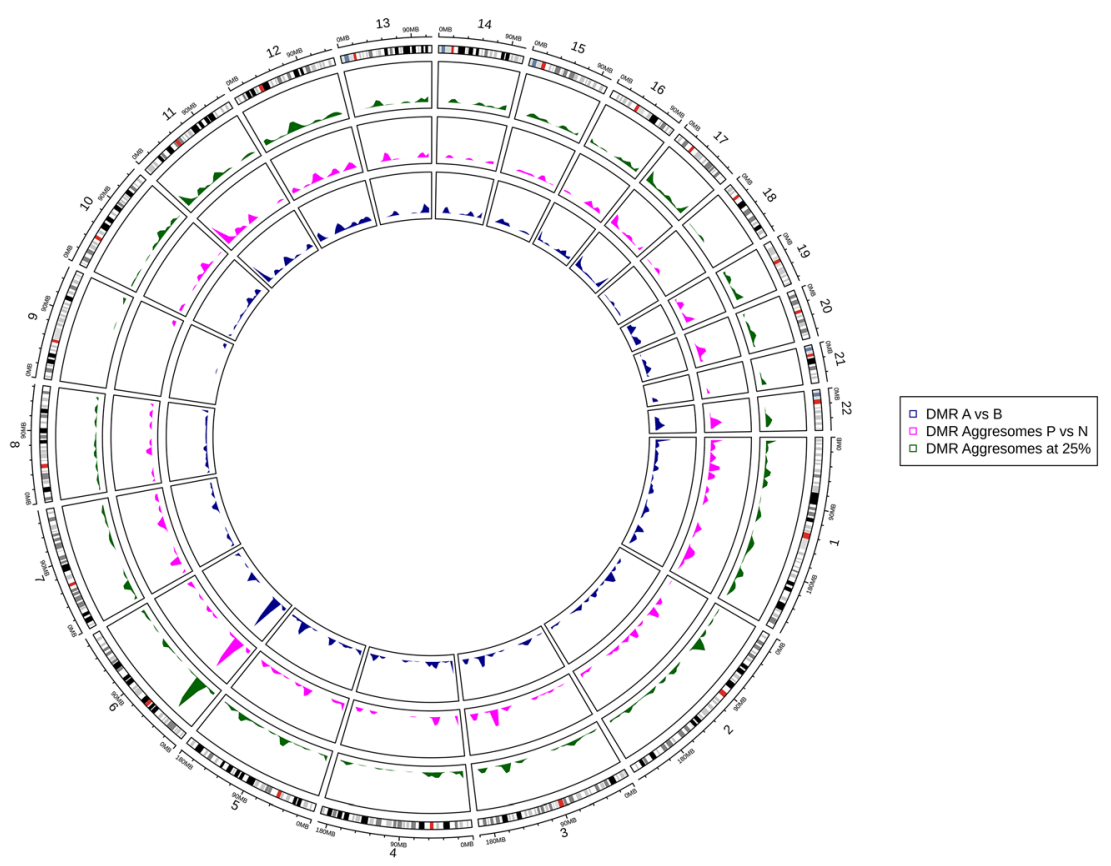

b

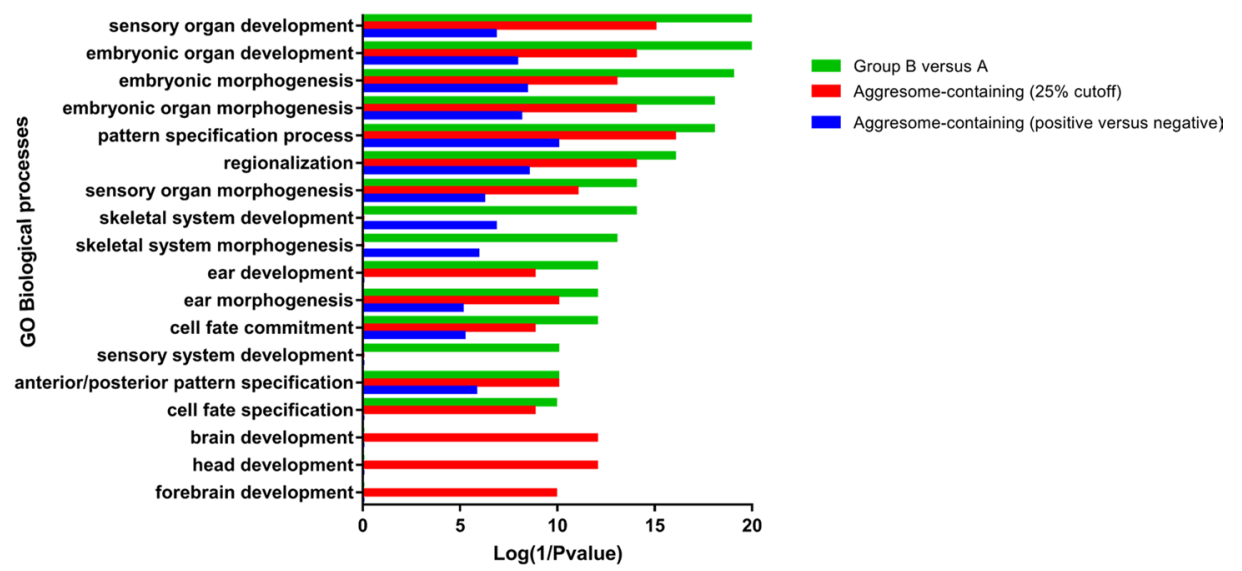

one subgroup, while ACPPs and CPPs distributed among both subgroups. The molecular stratification of CPTs had a significant impact on patient prognosis; however, the pathological stratification maintained the highest significance on patient outcomes. Despite having a lower significance than pathology, molecular classification identified ACPPs and CPPs with different biologic features similar to CPCs. The ACPPs are a challenging entity compared to CPPs and CPCs subgroups. Therefore, methylation-based classification provided a mean to segregate atypical tumors into good outcome (group A) or poor outcome (group B). In agreement with studies by other group [4, 5], methylation-based analysis was the most appropriate way to make the distinction. The overexpression of TP53 protein was observed only in the "B" molecular subgroup regardless of the pathological subtypes. Meanwhile, aggresomes were detected in all pathological and molecular subgroups with different percentages from negative up to $90 \%$ of tumor cells. The presence of aggresome had a nearly significant impact on the patient's outcome, while the $\geq 25 \%$ cutoff was comparable to the molecular classification in segregating samples into two subgroups, and to the pathological stratification in the prediction of patient outcomes. These results coincided with our previous study where $a \geq 20 \%$ aggresomes cutoff was a predictor of poor prognosis in the non-WNT/SHH molecular subgroup of pediatric MB [15]. The most commonly used model for assessing prognostic variables is multivariate survival analysis. Thereby, we 
a

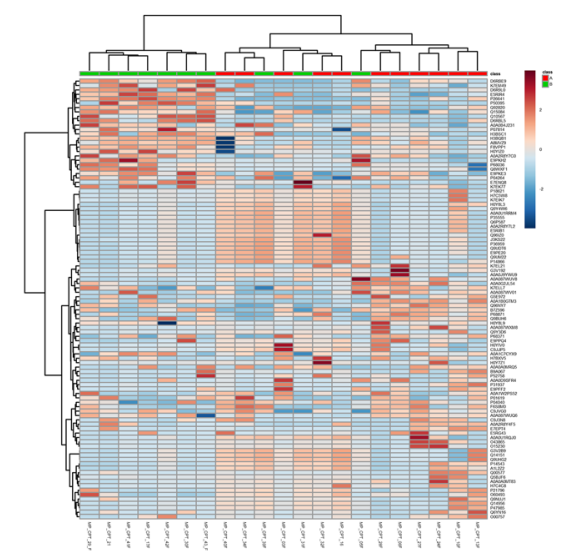

d

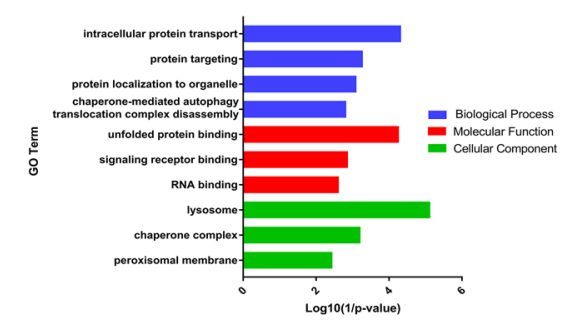

g

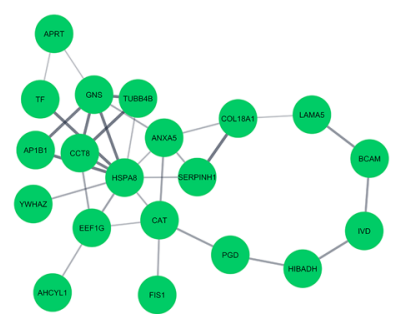

b

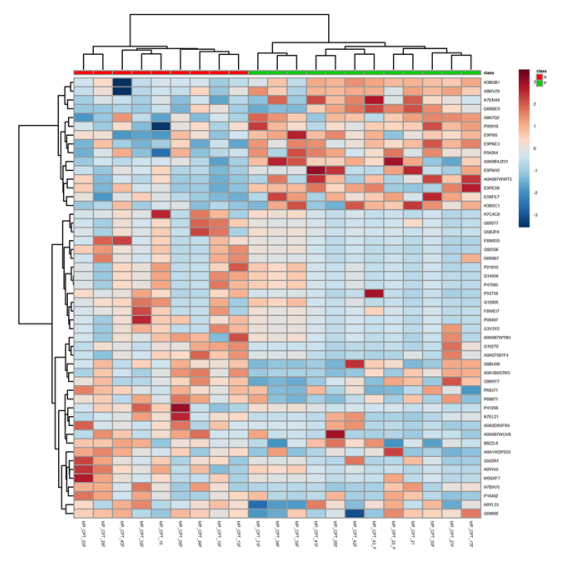

C

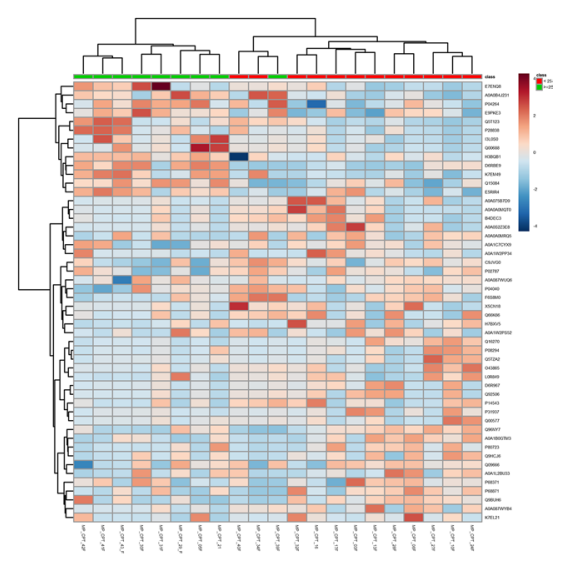

f

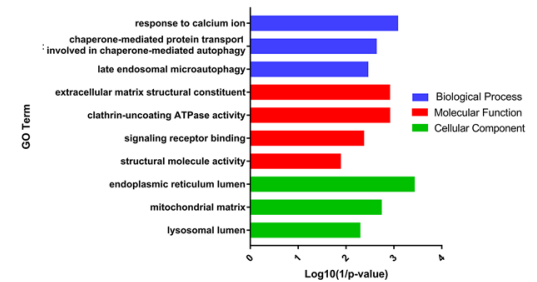

i
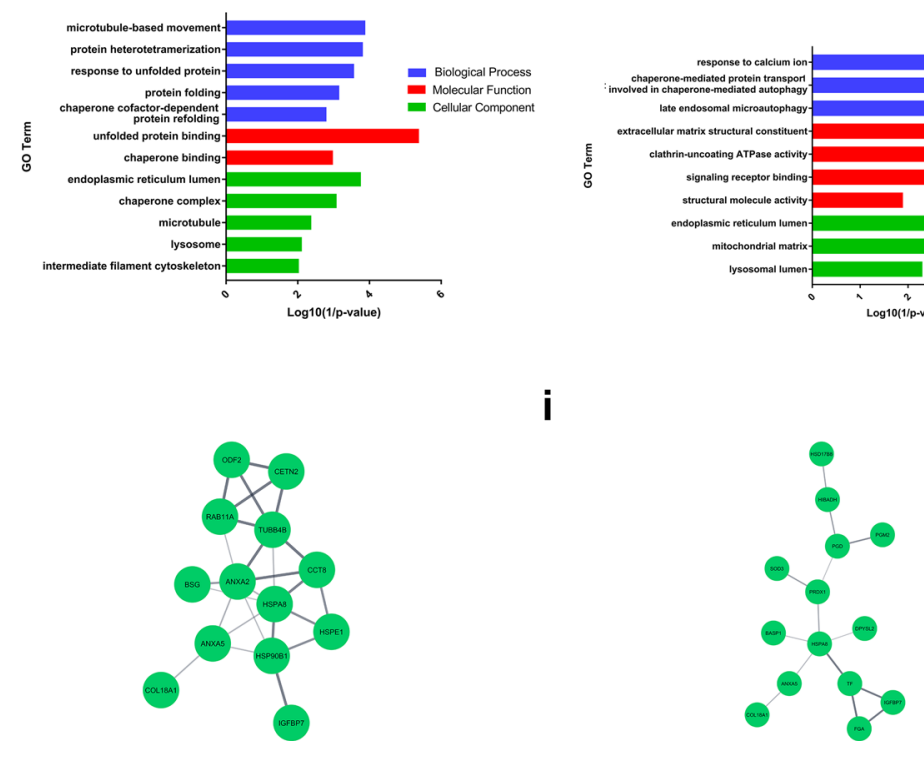

h
Fig. 5 Proteomic signature of CPTs. Hierarchical clustering heatmap of the top 50 significant DEPs ( $p$-values $<0.05)$ of a methylation-based classification, $\mathbf{b}$ aggresomes-positivity, and $\mathbf{c}$ the $\geq 25 \%$ aggresomes cutoff, with the y-axis represents samples analyzed and the $\mathrm{x}$-axis denotes Uniprot accessions. GO enrichment analysis of
DEPs of $\mathbf{d}$ methylation-based classification, $\mathbf{e}$ aggresomes-positivity, and $\mathbf{f}$ the $\geq 25 \%$ aggresomes cutoff. PPIs of interacting DEPs in $\mathbf{g}$ methylation-based classification, $\mathbf{h}$ aggresomes-positivity, and $\mathbf{i}$ the $\geq 25 \%$ aggresomes cutoff

genome-wide alterations we identified DMPs associated with aggresome formation in reference to the molecular subgroups. Various numbers of DMPs were associated with the three comparisons. The least number of DMPs in aggresome-based classification could be attributed to the fact that this comparison was based on a single biological feature; aggresomes. While the $25 \%$ cutoff applied more stringent criteria that increased the pathological and molecular uniformity of tumor populations. Interestingly, the methylation signature displayed by aggresome-positivity or the $25 \%$ aggresomes cutoff was the same as the methylationbased classification. Most of the enriched pathways were
To further explore the molecular signature of pediatric CPTs and examine the correlation of aggresomes with 
related to p53 binding, protein binding, folding, localization, or degradation in addition to the autophagy-related pathways such as mTOR signaling pathway and chaperone-mediated autophagy. Sholler et al. showed an activation of mTOR pathway in a chemo-resistant CPC patient and it was chosen for targeted therapy [37]. Therefore, further investigation of mTOR pathway in CPTs is warranted. Other pathways were enriched in DMPs including; pancreatic, colorectal, prostate, breast cancers as well as chronic and acute myeloid leukemias. All of these tumors had been reported to have aggresomes [10, 12, 13, 38-40], which implicates protein quality control in CPT pathogenesis. The manifestation of altered PQC signature that associated with DMPs was further confirmed by the enriched ATF6-mediated unfolded protein response in the DMRs. The enrichment analysis of DMRs revealed the TP53 signaling pathway which known to be involved in the development of CPTs $[6,37,41,42]$. The top significant DMR in the three comparisons comprised of ZICl and ZIC4 genes. Interestingly, DMPs annotated to those genes were hypermethylated in the "B" molecular subgroup and aggresomes positive tumors with positions either at the promoter sites or in the body of the gene. ZIC family genes are involved in a variety of developmental processes, including neurogenesis and morphogenesis [43]. Recently, ZICl gene was found to be silenced in colon cancer cell lines [44], primary colorectal cancer tissues [44], and gastric cancer [45]. Ectopic expression of ZIC1 suppressed cell proliferation and induced apoptosis through the MAPK and PI3K/ Akt pathways, as well as the $\mathrm{Bcl}-\mathrm{xl} / \mathrm{Bad} / \mathrm{Caspase} 3$ cascade $[44,45]$. As an important transcription factor, ZIC1 is essential to the regulation of Hedgehog signaling (Hh), Bone morphogenetic protein (BMP), and Notch signaling pathways in neural development [45, 46]. ZIC1 is also known to be interacting with GLI (glioma-associated oncogene homolog) genes, which function as both transcriptional activators and repressors downstream of the Shh signaling pathway and inhibitors of autophagy through the GLI2-PERK-eIF2 axis [47-49]. The hypermethylated signature of $\mathrm{ZICl}$ and the fact that $\mathrm{ZIC1}$ is negative regulators of SHH and Notch signaling pathways suggested the potential role of the ZIC1 in the pathogenesis of pediatric CPTs. This hypothesis could be supported by the detection of the notch signaling pathway in the enrichment analysis of DMRs and the hypomethylated signature of all DMPs that annotated to GLI2 gene in all comparisons. Accordingly, further examining the methylation status of ZICs transcription factors in the CPTs is required to establish their role in the pathogenesis of these tumors.

Proteomic analysis further supported the methylation signature, where most of the DEPs were related to heat shock proteins, ubiquitin, and the proteasome system. Enrichment analysis of both DMPs and DEPs showed similar biological functions, notably unfolded protein response, chaperone-mediated autophagy, late-endosomal microautophagy, protein binding, and cellular response to stress. Aggresomes-positivity expressed the highest number of DEPs and expressed the highest number of enriched pathways. This would suggest that aggresome-based classification reflects a biological fingerprint of CPTs.

The pathogenesis of pediatric CPTs is not well understood and the underlying mechanism of molecular alterations in these tumors remains unknown. In the current study, we examined the proteogenomic signatures of these tumors and identified potential targets that may help to understand these tumors through further analysis. We also defined aggresomes as a mechanism used by CPTs to achieve proteostasis. Aggresomes are thus not only a molecular prognostic marker but also a potential target for treatment of pediatric CPTs.

Acknowledgements We thank Dr. Mark W. Kieran for his valuable comments and discussions and Mai Mansour for her valuable editorial support.

Author contributions Conception and design: NA and SE. Pathological revision: HT. Laboratory experimentation: NA, NAS, AO and AS. Clinical data collection and analysis: NA, AM, NM, AY, ME, and MSZ. Data analysis and interpretation: NA, DH, MS, SM and SE. Manuscript writing: NA and SE. Final approval of manuscript: all authors.

Funding This work was funded by Egypt Cancer Network- USA (ECN) and JESOR grant (No. 8) from the Academy of Scientific Research and Technology (ASRT) in Egypt.

Data availability All data generated during this study are included in this article and its supplementary files. Raw methylation data were deposited at the Gene Expression Omnibus (GEO) under accession number GSE156090. The mass spectrometry proteomics data have been deposited to the ProteomeXchange Consortium via the PRIDE [1] partner repository with the dataset identifier PXD021076 and 10.6019/ PXD021076.

\section{Compliance with ethical standards}

Conflict of interest The authors declare no conflict of interest.

Ethical approval The IRB at CCHE has approved the waiver of patient consent form because the study used archived pathological samples and analysis performed on the samples does not affect patient well-being in any way. Patient confidentiality is maintained at all time in accordance with CCHE policies.

Open Access This article is licensed under a Creative Commons Attribution 4.0 International License, which permits use, sharing, adaptation, distribution and reproduction in any medium or format, as long as you give appropriate credit to the original author(s) and the source, provide a link to the Creative Commons licence, and indicate if changes were made. The images or other third party material in this article are included in the article's Creative Commons licence, unless indicated otherwise in a credit line to the material. If material is not included in the article's Creative Commons licence and your intended use is not 
permitted by statutory regulation or exceeds the permitted use, you will need to obtain permission directly from the copyright holder. To view a copy of this licence, visit http://creativecommons.org/licenses/by/4.0/.

\section{References}

1. Ogiwara H, Dipatri AJ, Alden TD et al (2012) Choroid plexus tumors in pediatric patients. Br J Neurosurg 26:32-37. https://doi. org/10.3109/02688697.2011.601820

2. Louis DN, Ohgaki H, Wiestler OD et al (2007) The 2007 WHO classification of tumours of the central nervous system. Acta Neuropathol 114:97-109. https://doi.org/10.1007/s00401-007-0243-4

3. Dudley RWR, Torok MR, Gallegos D et al (2015) Pediatric choroid plexus tumors: epidemiology, treatments, and outcome analysis on 202 children from the SEER database. J Neurooncol 121:201-207. https://doi.org/10.1007/s11060-014-1628-6

4. Merino DM, Shlien A, Villani A et al (2015) Molecular characterization of choroid plexus tumors reveals novel clinically relevant subgroups. Clin Cancer Res 21:184-192. https://doi. org/10.1158/1078-0432.CCR-14-1324

5. Thomas C, Sill M, Ruland V et al (2016) Methylation profiling of choroid plexus tumors reveals 3 clinically distinct subgroups. Neuro Oncol 18:790-796. https://doi.org/10.1093/neuonc/nov32 2

6. Dang L, Fan X, Chaudhry A et al (2006) Notch3 signaling initiates choroid plexus tumor formation. Oncogene 25:487-491. https:// doi.org/10.1038/sj.onc.1209074

7. Li L, Grausam KB, Wang J, Lun MP, Ohli J, Lidov HGW, Calicchio ML, Zeng E, Salisbury JL, Wechsler-Reya RJ, Lehtinen MK, Schüller U, Zhao H (2016) Sonic Hedgehog promotes proliferation of Notch-dependent monociliated choroid plexus tumour cells. Nat Cell Biol 25:289-313. https://doi.org/10.1016/j. bbi.2017.04.008

8. Chen B, Retzlaff M, Roos T, Frydman J (2011) Cellular strategies of protein quality control. Cold Spring Harb Perspect Biol 3:1-14. https://doi.org/10.1101/cshperspect.a004374

9. Johnston JA, Ward CL, Kopito RR (2012) A cellular response to misfolded proteins aggresomes . Cell 143:1883-1898

10. Simms-waldrip T, Rodriguez-Gonzalez A, Lin T et al (2008) Targeting the aggresome pathway in hematologic malignancies. Mol Genet Metab 94:283-286. https://doi.org/10.1016/j.ymgme 2008.03.012.Targeting

11. Moriya S, Komatsu S, Yamasaki K, Kawai Y (2015) Targeting the integrated networks of aggresome formation, proteasome, and autophagy potentiates ER stress-mediated cell death in multiple myeloma cells. Int J Oncol 46:474-486. https://doi.org/10.3892/ ijo.2014.2773

12. Nawrocki ST, Carew JS, Pino MS et al (2006) Aggresome disruption: a novel strategy to enhance bortezomib-induced apoptosis in pancreatic cancer cells. Cancer Res 66:3773-3781. https://doi. org/10.1158/0008-5472.CAN-05-2961

13. Miyahara K, Kazama H, Kokuba H et al (2016) Targeting bortezomib-induced aggresome formation using vinorelbine enhances the cytotoxic effect along with ER stress loading in breast cancer cell lines. Int J Oncol 49:1848-1858. https://doi.org/10.3892/ ijo.2016.3673

14. Nassar M, Samaha H, Ghabriel M et al (2017) LC3A silencing hinders aggresome vimentin cage clearance in primary choroid plexus carcinoma. Sci Rep 7:1-10. https://doi.org/10.1038/s4159 8-017-07403-5
15. Yehia M, Taha H, Salama A et al (2019) Association of aggresomes with survival outcomes in pediatric medulloblastoma. Sci Rep 9:1-10. https://doi.org/10.1038/s41598-019-49027-x

16. Wrede B, Hasselblatt M, Peters O et al (2009) Atypical choroid plexus papilloma: clinical experience in the CPT-SIOP-2000 study. J Neurooncol 95:383-392. https://doi.org/10.1007/s1106 0-009-9936-y

17. Aryee MJ, Jaffe AE, Corrada-Bravo H et al (2014) Minfi: A flexible and comprehensive bioconductor package for the analysis of Infinium DNA methylation microarrays. Bioinformatics 30:13631369. https://doi.org/10.1093/bioinformatics/btu049

18. Maksimovic J, Phipson B, Oshlack A (2016) A cross-package Bioconductor workflow for analysing methylation array data. F1000Research 5:1281. https://doi.org/10.12688/f1000resea rch.8839.1

19. Teschendorff AE, Menon U, Gentry-Maharaj A et al (2009) An epigenetic signature in peripheral blood predicts active ovarian cancer. PLoS ONE. https://doi.org/10.1371/journal.pone.0008274

20. Johnson WE, Li C, Rabinovic A (2007) Adjusting batch effects in microarray expression data using empirical Bayes methods. Biostatistics 8:118-127. https://doi.org/10.1093/biostatistics/kxj037

21. McCartney DL, Walker RM, Morris SW et al (2016) Identification of polymorphic and off-target probe binding sites on the Illumina Infinium MethylationEPIC BeadChip. Genomics Data 9:22-24. https://doi.org/10.1016/j.gdata.2016.05.012

22. Pidsley R, Zotenko E, Peters TJ et al (2016) Critical evaluation of the Illumina MethylationEPIC BeadChip microarray for wholegenome DNA methylation profiling. Genome Biol 17:1-17. https ://doi.org/10.1186/s13059-016-1066-1

23. Van Der Maaten L (2015) Accelerating t-SNE using tree-based algorithms. J Mach Learn Res 15:3221-3245

24. Capper D, Jones DTW, Sill M et al (2018) DNA methylationbased classification of central nervous system tumours. Nature 555:469-474. https://doi.org/10.1038/nature26000

25. Smyth GK (2004) Linear models and empirical bayes methods for assessing differential expression in microarray experiments. Stat Appl Genet Mol Biol. https://doi.org/10.2202/1544-6115.1027

26. Jaffe AE, Murakami P, Lee $\mathrm{H}$ et al (2012) Bump hunting to identify differentially methylated regions in epigenetic epidemiology studies. Int J Epidemiol 41:200-209. https://doi.org/10.1093/ije/ dyr238

27. Gu Z, Gu L, Eils R et al (2014) Circlize implements and enhances circular visualization in R. Bioinformatics 30:2811-2812. https:// doi.org/10.1093/bioinformatics/btu393

28. Chen J, Bardes EE, Aronow BJ, Jegga AG (2009) ToppGene Suite for gene list enrichment analysis and candidate gene prioritization. Nucleic Acids Res 37:305-311. https://doi.org/10.1093/nar/gkp42 7

29. Mclean CY, Bristor D, Hiller M et al (2010) GREAT improves functional interpretation of cis-regulatory regions HHS public access author manuscript. Nat Biotechnol 28:495-501. https:// doi.org/10.1038/nbt.1630

30. Zhou G, Soufan O, Ewald J et al (2019) NetworkAnalyst 3.0: a visual analytics platform for comprehensive gene expression profiling and meta-analysis. Nucleic Acids Res 47:W234-W241. https://doi.org/10.1093/nar/gkz240

31. Stacklies W, Redestig H, Scholz M et al (2007) pcaMethodsA bioconductor package providing PCA methods for incomplete data. Bioinformatics 23:1164-1167. https://doi.org/10.1093/bioin formatics/btm069

32. Gulcicek EE, Colangelo CM, McMurray W, Stone K, Williams K, Wu T, Zhao H, Spratt H, Kurosky A, Wu B (2005) Proteomics and the analysis of proteomic data : an overview of current protein-profiling technologies. Curr Protoc Bioinform. https://doi. org/10.1002/0471250953.bi1301s10 
33. Dieterle F, Ross A, Senn H (2006) Probabilistic quotient normalization as robust method to aacount for dilution of complex biuological mixtures. Anal Chem 78:4281-4290

34. Soudy M, Anwar A, Ahmed EA, Osama A (2019) UniprotR: retrieving and visualizing protein sequence and functional information from universal protein resource (UniProt knowledgebase). J Proteomics 213:103613. https://doi.org/10.1016/j.jprot .2019 .103613

35. Shannon P, Markiel A, Ozier O, Baliga NS, Wang JT, Ramage $D$ et al (1971) Cytoscape: a software environment for integrated models. Genome Res 13:426. https://doi.org/10.1101/gr.12393 03.metabolite

36. Riley RD, Snell KIE, Ensor J et al (2019) Minimum sample size for developing a multivariable prediction model: PART II — binary and time-to-event outcomes. Stat Med 38:1276-1296. https://doi. org/10.1002/sim.7992

37. Cornelius A, Foley J, Bond J et al (2017) Molecular guided therapy provides sustained clinical response in refractory choroid plexus carcinoma. Front Pharmacol. https://doi.org/10.3389/fphar 2017.00652

38. Papandreou CN, Logothetis CJ (2004) Bortezomib as a potential treatment for prostate cancer. Cancer Res 64:5036-5043. https:// doi.org/10.1158/0008-5472.CAN-03-2707

39. Brodsky JL (2017) Targeting protein quality control pathways in breast cancer. BMC Biol 15:1-20. https://doi.org/10.1186/s1291 5-017-0449-4

40. Dingli D, Ailawadhi S, Bergsagel PL et al (2017) Therapy for relapsed multiple myeloma: guidelines from the mayo stratification for myeloma and risk-adapted therapy. Mayo Clin Proc 92:578-598

41. Ma X, Drannik A, Jiang F et al (2017) Crosstalk between Notch and Sonic hedgehog signaling in a mouse model of amyotrophic lateral sclerosis. NeuroReport 28:141-148. https://doi. org/10.1097/WNR.0000000000000725
42. Wang J, Merino DM, Light N et al (2019) Mycand loss of p53 cooperate to drive formation of choroid plexus carcinoma. Cancer Res 79:2208-2219. https://doi.org/10.1158/0008-5472. CAN-18-2565

43. Gaston-Massuet C, Henderson DJ, Greene NDE, Copp AJ (2005) Zic4, a zinc-finger transcription factor, is expressed in the developing mouse nervous system. Dev Dyn 233:1110-1115. https:// doi.org/10.1002/dvdy.20417

44. Gan L, Chen S, Zhong J et al (2011) ZIC1 is downregulated through promoter hypermethylation, and functions as a tumor suppressor gene in colorectal cancer. PLoS ONE. https://doi. org/10.1371/journal.pone.0016916

45. Wang LJ, Jin HC, Wang X et al (2009) ZIC1 is downregulated through promoter hypermethylation in gastric cancer. Biochem Biophys Res Commun 379:959-963. https://doi.org/10.1016/j. bbrc.2008.12.180

46. Zhong J, Chen S, Xue M et al (2012) ZIC1 modulates cell-cycle distributions and cell migration through regulation of sonic hedgehog, PI3K and MAPK signaling pathways in gastric cancer. BMC Cancer 12:1-10. https://doi.org/10.1186/1471-2407-12-290

47. Mizugishi K, Aruga J, Nakata K, Mikoshiba K (2001) Molecular properties of Zic proteins as transcriptional regulators and their relationship to GLI proteins. J Biol Chem 276:2180-2188. https ://doi.org/10.1074/jbc.M004430200

48. Jimenez-Sanchez M, Menzies FM, Chang YY et al (2012) The Hedgehog signalling pathway regulates autophagy. Nat Commun 3:1200-1211. https://doi.org/10.1038/ncomms2212

49. Milla LA, González-Ramírez CN, Palma V (2012) Sonic hedgehog in cancer stem cells: a novel link with autophagy. Biol Res 45:223-230. https://doi.org/10.4067/S0716-97602012000300004

Publisher's Note Springer Nature remains neutral with regard to jurisdictional claims in published maps and institutional affiliations.

\section{Authors and Affiliations}

\section{Nada Amer ${ }^{1} \cdot$ Hala Taha ${ }^{2,3}$. Dina Hesham ${ }^{1} \cdot$ Nouran Al-Shehaby $^{1} \cdot$ Amal Mosaab $^{1} \cdot$ Mohamed Soudy $^{4} \cdot$ Aya Osama $^{4}$. Noura Mahmoud ${ }^{5}$. Moatasem Elayadi ${ }^{5,6}$. Ayda Youssef ${ }^{7,8}$. Mohamed Elbeltagy ${ }^{9,10}$ - Mohamed Saad Zaghloul ${ }^{11,12}$. Sameh Magdeldin ${ }^{4,13}$ (1) Ahmed A. Sayed ${ }^{14,15}$. Shahenda El-Naggar ${ }^{1}$ (])}

1 Tumor Biology Research Program, Basic Research Unit, Research Department, Children's Cancer Hospital Egypt, 1 Sekket El Emam, El Madbah El Kadeem Yard, Sayeda Zeinab, Cairo 57357, Egypt

2 Department of Pathology, Children's Cancer Hospital Egypt, Cairo 57357, Egypt

3 Department of Pathology, National Cancer Institute, Cairo University, Cairo, Egypt

4 Proteomics and Metabolomics Research Program, Basic Research Unit, Research Department, Children's Cancer Hospital Egypt, Cairo 57357, Egypt

5 Department of Pediatric Oncology, Children's Cancer Hospital Egypt, Cairo 57357, Egypt

6 Department of Pediatric Oncology, National Cancer Institute, Cairo University, Cairo, Egypt

7 Department of Radiology, Children's Cancer Hospital Egypt, Cairo 57357, Egypt

8 Department of Radiology, National Cancer Institute, Cairo University, Cairo, Egypt
9 Department of Neurosurgery, Children's Cancer Hospital Egypt, Cairo 57357, Egypt

10 Department of Neurosurgery, Faculty of Medicine, Cairo University, Cairo, Egypt

11 Department of Radiotherapy, National Cancer Institute, Cairo University, Cairo, Egypt

12 Department of Radiotherapy, Children's Cancer Hospital Egypt, Cairo 57357, Egypt

13 Department of Physiology, Faculty of Veterinary Medicine, Suez Canal University, Ismailia, Egypt

14 Genomics Research Program, Basic Research Unit, Research Department, Children's Cancer Hospital Egypt, Cairo 57357, Egypt

15 Department of Biochemistry, Faculty of Science, Ain Shams University, Cairo, Egypt 